\title{
Evaluación de la calidad del agua en el embalse hidroeléctrico El Quimbo $^{1}$
}

\section{Water quality evaluation in the El Quimbo hydroelectric dam}

\author{
Losada, L.C., Rueda-Sanabria, C.A. y Martínez, P.
}

Recibido: agosto 12 de 2019 - Aceptado: junio 20 de 2020

\begin{abstract}
${ }^{1}$ Resumen-Los embalses construidos para las hidroeléctricas además de proveer energía, también son reservorios de agua para consumo humano, animal y riego en cultivos agrícolas, por tal motivo es importante evaluar la calidad de estas aguas periódicamente. Este estudio diagnosticó la calidad de agua del embalse El Quimbo usando el Índice Simplificado de Calidad del Agua (ISQA) el cual contempla la medición oxígeno disuelto (OD), conductividad, temperatura, demanda química de oxígeno (DQO) y sólidos suspendidos totales (SST). Se tomaron muestras en siete puntos del embalse, tres puntos a lo largo del embalse y cuatro en los cuerpos de agua que surten el reservorio, esto durante seis salidas de campo, desde mayo hasta octubre de 2017. Así mismo, en los tres puntos del embalse se tomaron muestras a diferentes profundidades $(0.1,5.0$ y $10.0 \mathrm{~m})$. Los resultados obtenidos a partir de los análisis fisicoquímicos descritos anteriormente mostraron que en promedio el agua del embalse es de buena calidad, con potencial uso para la pesca, recreación, y no aptas para consumo humano sin tratamiento convencional; por otro lado, el agua de los afluentes se calificó en promedio como de mediana calidad, destacando la quebrada Guandinosa, la cual presentó el valor más
\end{abstract}

${ }^{1}$ Producto derivado del proyecto de investigación "Determinación de la calidad de agua del embalse El Quimbo utilizando el índice simplificado de calidad de agua (ISQA)". Presentado por el Grupo de Investigación Efecto Ambiental, de la Corporación Universitaria del Huila - Corhuila en la Octava convocatoria interna de proyectos de investigación, proyección social y jóvenes investigadores, Resolución $\mathrm{N}^{\circ} 1524$ de 2017.

L.C. Losada, Corporación Universitaria del Huila - Corhuila, Neiva Colombia, email: luis.losada@ corhuila.edu.co

C.A. Rueda-Sanabria, Corporación Universitaria del Huila - Corhuila, Neiva Colombia, email: carlos.rueda@ corhuila.edu.co

P. Martínez, Corporación Universitaria del Huila - Corhuila, Neiva Colombia, email: paula.martinez@corhuila.edu.co.

Como citar este artículo: Losada, L. C., Rueda-sanabria, C. A., Martínez, P. Evaluación de la calidad del agua en el embalse hidroeléctrico El Quimbo, Entre Ciencia e Ingeniería, vol. 14, no. 27, pp. 107-116, enero-junio 2020. DOI: https://doi.org/10.31908/19098367.1800.

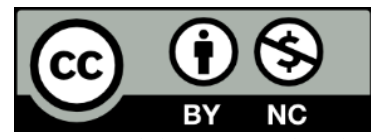

Attribution-NonCommercial 4.0 Intenational (CC By-NC 4.0) bajo de ISQA (35.2), clasificando su uso para recreación, riego y uso industrial.

Palabras clave - Embalse, índice simplificado de la calidad del agua, ISQA, parámetros fisicoquímicos, Quimbo, ríos.

Abstract-Dams are built to provide energy, to act as water reservoirs for human and animal consumption, and to water cultivated fields; for those reasons, it is of high importance to assess their water quality periodically. This research diagnosed the quality of water of the Quimbo Dam by using the Simplified Index of Water Quality (SIWQ), which contemplates the measure of dissolved oxygen (DO), conductivity, temperature, Chemical demand of oxygen (QDO) and total suspended solids (TSS). Samples were taken in seven different points along the dam, and four points on rivers that tribute their waters to the dam, during field trips from May until October, 2017. In the same way, in each point, samples were taken at different depths $(0.1,5.0$, and 10.0 meters). The obtained results from the analysis of physicochemical parameters described above showed that in average the water quality of Quimbo Dam is in a good condition, with potential for fishing and recreational activities; it is not apt for human consumption without conventional treatment; on the other hand, the water from the tributaries showed medium quality, specially Guandinosa stream which presented the lowest value of SIWQ (35.2), classified as useful only for recreation, water for cultivated fields and industrial activities.

Keywoeords- Dam, simplified water quality index, ISQA, physical-chemical parameters, Quimbo, rivers.

\section{NOMENCLATURA}

CE: conductividad eléctrica, DBO: demanda bioquímica de oxígeno, DQO: demanda química de oxígeno, OD: oxígeno disuelto, SST: sólidos suspendidos totales.

\section{INTRODUCCIÓN}

GL desarrollo de los asentamientos humanos ha estado muy Cligado a la posibilidad de conseguir agua para todo tipo de actividad antropogénica, desde el simple aseo personal, la preparación de alimentos, procesos agrícolas y los diferentes procesos industriales, por ello el afán del hombre de construir reservorios que garanticen el abastecimiento del recurso 
hídrico [1]-[6]. Uno de estos reservorios son los embalses hidroeléctricos, donde se aprovecha la energía mecánica de los ríos, para ser convertida en energía eléctrica. El beneficio es indiscutible, producción de electricidad, nuevas líneas económicas como proyectos piscícolas y turísticos, sin embargo, cada una de estas actividades consume gran cantidad de agua produciendo un impacto ambiental negativo sobre los cuerpos de agua [3], causando desviación y fragmentación de los causes de los ríos, a su vez produce bloqueo del movimiento de animales que viven en estos hábitats [7], [8], disminución de caudales necesarios para los ciclos vitales de peces aguas abajo del sitio de descarga [9], se ve afectada la biodiversidad, aumento de peces invasores y disminución de peces nativos tanto aguas arriba como aguas debajo de una represa [10], [11]. Se puede producir erosión del suelo de las laderas del embalse por el desprendimiento de minerales y materia orgánica, aumentando la sedimentación [12], [13], procesos de eutrofización [14] que generan cambios en los niveles de oxígeno disuelto (OD) [15], variaciones en el pH, temperatura; adicional, sobre los cuerpos de agua que surten los embalses se vierten aguas residuales industriales y domésticas cargadas de microorganismos patógenos resistentes a diversos antibióticos, aumentando su carga contaminante [14], [16]. Evaluar la calidad de estas aguas es preponderante para determinar su posible uso, la salud de los ecosistemas acuáticos, y la toma de decisiones frente al control y mitigación del riesgo sanitario [17]-[20]. Existen diversas maneras de evaluar la calidad del agua a través de indicadores ambientales físicos, químicos y biológicos, los cuales reciben el nombre de Índices de Calidad del Agua (ICA) [17]. En 1965 Horton desarrolló el primer ICA, en el cual se estiman condiciones de contaminación acuática a partir los parámetros $\mathrm{pH}$, conductividad, DO, alcalinidad, contenido de cloruros, temperatura $\left(\Delta \mathrm{t},{ }^{\circ} \mathrm{C}\right)$, recuento de coliformes [21]. Luego, en 1970 se propuso El índice general de calidad de agua, en este se evalúa la calidad del agua para consumo humano teniendo en cuenta el análisis de OD, coliformes fecales, $\mathrm{pH}$, demanda biológica de oxígeno $\left(\mathrm{DBO}_{5}\right)$, contenido de nitratos, fosfatos totales, temperatura $\left(\Delta \mathrm{t},{ }^{\circ} \mathrm{C}\right)$, turbiedad y sólidos totales [22]. A partir de ahí se continuó en la adaptación, mejoramiento y desarrollo de otros ICAs [23]. Uno de los índices de mejor aplicabilidad a estudios de calidad de agua en sistemas lénticos (embalses, lagos) y lóticos (ríos) es el Índice Simplificado de Calidad de Agua (ISQA) desarrollado en España por Queralt en 1982, el cual opera con solo cinco parámetros temperatura $\left(\Delta \mathrm{t},{ }^{\circ} \mathrm{C}\right), \mathrm{OD}$, conductividad eléctrica (CE), DQO y sólidos suspendidos totales (SST). El ISQA permite obtener resultados garantizados, minimizando tiempo y recursos [24]-[29]. El ISQA se obtiene a partir de la ecuación (1) como se pudo encontrar en [3], [24], [30].

$$
I S Q A=E(A+B+C+D)
$$

Donde $E$ es un factor que depende de la temperatura, $A$ es el factor dependiente de la DQO, $B$ depende de los SST, $C$ es el factor que depende del OD y $D$ depende de la CE.

En la Tabla 1 se pueden observar las condiciones para calcular cada uno de los factores que se deben tener en cuenta para hallar el índice ISQA [24], [30]. Este índice permite definir un criterio cualitativo de la calidad del agua y a partir de esto asignar un posible uso, como se puede observar en la Tabla I [31].

El embalse hidroeléctrico el Quimbo se instaló en las aguas del río Magdalena que pasan por el centro del departamento del Huila, entre los municipios de Garzón, Gigante, Agrado, Altamira, Paicol y Tesalia (Fig. 1). Genera una potencia media de $2.216 \mathrm{GWh} / \mathrm{año}$, por lo cual cubre aproximadamente el $4 \%$ de la demanda energética de Colombia [32].

TABLA I

CLASIFICACIÓN DEL AGUA SEGÚN EL ISQA [31]

\begin{tabular}{|c|c|c|}
\hline Índice ISQA & Clasificación & Aptitudes de uso \\
\hline $85-100$ & Excelente calidad & Todos los usos \\
\hline $60-85$ & Buena calidad & $\begin{array}{c}\text { Agua potable (Tratamiento } \\
\text { convencional), pesca y uso } \\
\text { recreativo }\end{array}$ \\
\hline $45-60$ & Mediana calidad & $\begin{array}{c}\text { Riego hortícola, agua } \\
\text { industrial y agua potable } \\
\text { (tratamiento especial) }\end{array}$ \\
\hline $30-45$ & Mala calidad & $\begin{array}{c}\text { Riego de plantas sin } \\
\text { consumo directo }\end{array}$ \\
\hline$<30$ & Muy mala calidad & $\begin{array}{c}\text { Navegación, refrigeración. } \\
\text { Uso muy restringido }\end{array}$ \\
\hline
\end{tabular}

El presente estudio tiene como objetivo diagnosticar el estado de la calidad del agua de la central hidroeléctrica el Quimbo por medio del ISQA, debido al impacto ambiental negativo que genera el material vegetal talado en la zona del embalse, el cual no fue retirado a tiempo y terminó depositado en el embalse.

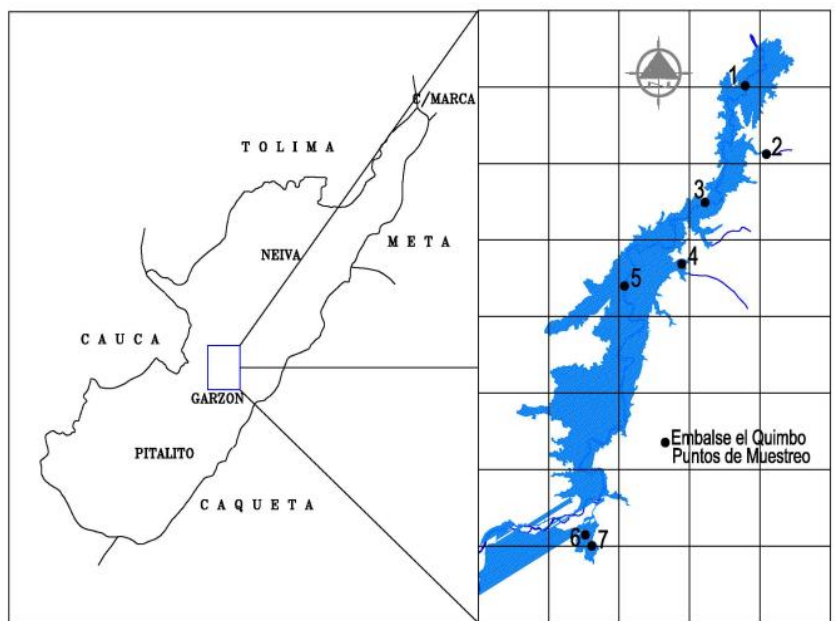

Fig. 1. Ubicación geográfica: Izquierda. Mapa del departamento del Huila, se muestra ubicación del embalse en rectángulo; Derecha. Rectángulo ampliado puntos de muestreo en el embalse el Quimbo, compuertas Quimbo (1), quebrada Guandinosa (2), Gigante acostado (3), río Loro (4), la Trina (5), inicio del embalse (6) y quebrada Garzón (7).

\section{MetodologíA}

\section{A. Puntos de muestreo}

Para los puntos de muestreo se tomó como referencia el estudio realizado en la hidroeléctrica de Copa en Boyacá [14] y un estudio realizado sobre los ecosistemas acuáticos de la cuenca alta del río Magdalena [32]. Establecimos siete puntos 
de muestreo cómo se pueden observar en la Fig. 1 y en Tabla II, cuatro corresponden a puntos dentro del embalse (1, $3,5$ y 6$)$ y tres en los afluentes al embalse $(2,4$ y 7$)$. Se realizaron seis campañas o salidas de campo, llevándose a cabo una mensual (de mayo a octubre).

TABLA II

GEORREFERENCIACIÓN DE LOS PUNTOS DE MUESTREO

\begin{tabular}{|c|c|c|c|}
\hline $\begin{array}{c}\text { Punto de } \\
\text { muestreo }\end{array}$ & $\begin{array}{c}\text { Ubicación en } \\
\text { Fig. 1-B }\end{array}$ & Latitud & Longitud \\
\hline $\begin{array}{c}\text { Compuertas } \\
\text { Quimbo }\end{array}$ & 1 & $2^{\circ} 25^{\prime} 30.4^{\prime \prime}$ & $75^{\circ} 34^{\prime} 12.7^{\prime \prime}$ \\
\hline $\begin{array}{c}\text { Quebrada } \\
\text { Guandinosa }\end{array}$ & 2 & $2^{\circ} 23^{\prime} 13.3^{\prime \prime}$ & $75^{\circ} 33^{\prime} 55^{\prime \prime}$ \\
\hline $\begin{array}{c}\text { Gigante } \\
\text { acostado }\end{array}$ & 3 & $2^{\circ} 21^{\prime} 23.6^{\prime \prime}$ & $75^{\circ} 35^{\prime} 48.8^{\prime \prime}$ \\
\hline Río Loro & 4 & $2^{\circ} 18^{\prime} 21.9^{\prime \prime}$ & $75^{\circ} 38^{\prime} 45.2^{\prime \prime}$ \\
\hline \begin{tabular}{c} 
La Trina \\
\hline $\begin{array}{c}\text { Inicio del } \\
\text { embalse }\end{array}$
\end{tabular} & 5 & $2^{\circ} 18^{\prime} 53.48^{\prime \prime}$ & $75^{\circ} 37^{\prime} 28.00^{\prime \prime}$ \\
\hline $\begin{array}{c}\text { Quebrada } \\
\text { Garzón }\end{array}$ & 7 & $2^{\circ} 13^{\prime} 23.8^{\prime \prime}$ & $75^{\circ} 39^{\prime} 15^{\prime \prime}$ \\
\hline
\end{tabular}

\section{B. Toma de muestras y determinación de los parámetros fisicoquímicos}

Se tomaron muestras de agua dentro del embalse a tres profundidades, la primera a $0.1 \mathrm{~m}$, la segunda a $5 \mathrm{~m}$ y la tercera a $10 \mathrm{~m}$, tratando de cubrir la columna de agua. Para llevar a cabo este proceso, se utilizó una botella muestreadora vertical tipo Van Dorn de 2.2 L (biológika, COL) siguiendo el protocolo consignado en [33]. La toma de muestras en los tres afluentes se hizo a $100 \mathrm{~m}$ antes de su desembocadura. Los datos fisicoquímicos tomados en campo como la temperatura $(\Delta t$, $\left.{ }^{\circ} \mathrm{C}\right)$, OD, $\mathrm{pH}$ y conductividad se realizaron con un multiparámetro portátil YSI 556 MPS (YSI-Xylem, EUA) siguiendo los protocolos propuestos por el IDEAM [33]-[36] respectivamente. En la Determinación de DQO, se tomaron alícuotas de $3 \mathrm{~mL}$, se digirieron con el kit Cell Test HC 613856 (Merck, USA). Las reacciones de digestión se llevaron a cabo en un termorreactor de baño seco Wealtec-HB-1 (Wealtec, USA) durante $120 \mathrm{~min}$ a $148{ }^{\circ} \mathrm{C}$. Las muestras digeridas se leyeron en un espectrofotómetro portátil LaMotte 410 COD3 plus (LaMotte, USA). Los valores de DQO se obtuvieron siguiendo el protocolo del IDEAM [37], y el método 5220-D del Standar Methods for the Examination of Water and Wastewater [38]. Para obtener el dato SST se usaron filtros de fibra de vidrio de $25 \mathrm{~mm}$ de diámetro y 0.7 $\mathrm{mm}$ de grosor (VWR-Avantor, EUA) y se llevaron a un horno Memmert UNB200 (Memmert, Alemania) a $103{ }^{\circ} \mathrm{C}$ por dos horas. Para medir la masa de los filtros antes y después de la filtración se usó una balanza analítica Radwag AS 220.E2 (Radwag, Polonia). Esto se hizo siguiendo el protocolo del IDEAM [39] y el método 2540-D del Standar Methods for the Examination of Water and Wastewater [38]. Para la $\mathrm{DBO}_{5}$, se empleó el protocolo descrito en el método 5210-D del Standar Methods for the Examination of Water and Wastewater [38].
Cada una de las muestras se analizó por triplicado. El volumen de muestra a sembrar y la cantidad de agua de dilución en cada botella Winkler se determinaron según la tabla establecida por el IDEAM [40]. Las muestras para $\mathrm{DBO}_{5}$ se mantuvieron en una incubadora Memmert IPP110 (Memmert, Alemania) a 37 ${ }^{\circ} \mathrm{C}$. El OD inicial y final se midió con un multiparámetro de mesa WTW multi 9319 IDS (WTW-Xylem, Alemania) y sonda OD WTW FDO 925 (WTW-Xylem, Alemania).

Para calcular el índice simplificado de calidad de agua ISQA se utilizó la ecuación (1). Una vez se obtuvo el índice ISQA para cada punto de muestreo, se clasificó según su calidad.

\section{ANÁLISIS DE RESULTADOS}

Durante las campañas realizadas se evidenciaron afectaciones negativas al ambiente por parte del proyecto hidroeléctrico y de los pobladores de los municipios que rodean al embalse. En el punto quebrada Garzón se pudo apreciar una fuerte afectación a este ecosistema, debido a los residuos sólidos que son arrojados desde el casco urbano del municipio de Garzón a esta quebrada. Además, en la desembocadura de la quebrada al embalse se observa estancamiento de las aguas, depósito y descomposición de la madera que fue arrastrada durante la inundación del Quimbo, lo que produce malos olores y presencia de espuma flotante. De igual forma, se pudo evidenciar un alto crecimiento de la planta Eichhornia crassipes (buchón de agua) producto de la eutrofización de las aguas. El otro punto de evidente afectación fue en la desembocadura del río Loro al embalse, en este punto se pudo contemplar un paisaje modificado por la inundación del embalse, se encontró troncos de árboles talados de gran tamaño, plantas herbáceas de y plantas no nativas. Así mismo, se pudo apreciar que en comparación con la quebrada Garzón su corriente se veía afectada por algún tipo de residuo sólido antropogénico. A continuación, se hará un análisis de cada uno de los parámetros fisicoquímicos evaluados en el embalse el Quimbo, y luego se analizarán los resultados de la calidad del agua en cada uno de los puntos de muestreo.

\section{A. Análisis de la temperatura}

De acuerdo con los resultados obtenidos en los seis meses de muestreo en el embalse el Quimbo (Tabla III), se registró una temperatura promedio de $26.4{ }^{\circ} \mathrm{C}$. Se pudo determinar que en el mes de agosto se registró las temperaturas más altas en los puntos de La Trina, Gigante acostado y compuertas del embalse el Quimbo, con una temperatura promedio superficial de $28.4{ }^{\circ} \mathrm{C}$. Esto puede ser debido a que estos puntos están ubicados en el centro y parte norte del embalse, donde recibe una mayor radiación solar, por su gran espejo de agua y no hay una gran movilidad de estas aguas. Según el IDEAM la temperatura ambiental promedio del mes de agosto de 2017 en el departamento del Huila fue de $29.7^{\circ} \mathrm{C}$, en el mismo mes del año 2016 se registró una temperatura promedio de $30^{\circ} \mathrm{C}$, y un promedio histórico de los meses de mayo del periodo comprendido entre los años 1981 a 2010 se registró un valor aproximado de $29{ }^{\circ} \mathrm{C}$. Sumado a lo anterior, el registro de precipitaciones del mes de agosto fue tan solo de $30 \mathrm{~mm}$, lo 
cual nos muestra que es temporada de sequía y temperaturas elevadas [41]. Una posible razón por la cual en el inicio del embalse registró los valores más bajos en la temperatura, es porque en ese lugar todavía influye la corriente que viene desde el sur por el rio Magdalena y por el rio Suaza, haciendo que la temperatura en este lugar sea más baja. En los tres afluentes que se analizaron q. Guandinosa, q. Garzón y r. Loro se presentó un comportamiento normal de la temperatura y es acorde con los resultados obtenidos en un estudio realizado por EMGESA [32].

\section{B. Análisis del oxígeno disuelto $(O D)$}

Los resultados de OD tuvieron una gran variabilidad en todas las campañas (Tabla IV). En cuanto a los puntos de muestreo sobre el Quimbo, se encontró que el mes que presentó el mayor valor de OD fue mayo, en el punto inicio del embalse con un valor de $7.04 \mathrm{mg} / \mathrm{L}$, siendo un dato óptimo; mientras que en el mes de septiembre se registró el OD más bajo, con un valor de $3.72 \mathrm{mg} / \mathrm{L}$. Una posible razón de este valor registrado es porque en ese lugar todavía tiene gran influencia la corriente del río Magdalena.

En general en los demás puntos de muestreo el OD registrado estuvo por debajo de los $6 \mathrm{mg} / \mathrm{L}$. Sin embargo, no se evidenció relación alguna con la variación de la temperatura en los puntos de muestreo. Estos datos indican que el agua puede ser utilizada para actividades de piscicultura, debido a que los resultados a 0.1 metros y 5 metros, contienen el oxígeno disuelto necesario para que se pueda ejercer esta actividad [42]. Por otro lado, teniendo en cuenta la columna de agua y a medida que se aumentaba en la profundidad de las aguas del Quimbo, se encontró que el OD iba disminuyendo, esto debido a la estratificación normal de los cuerpos de agua lénticos. El OD medido sobre los afluentes mostró que en el r. Loro se registró los mayores valores para este parámetro, seguido de la q. Guandinosa y con más bajos valores en la q. Garzón, donde se evidenció el deterioro del ecosistema con presencia de un aumento de plantas acuáticas. Para todos los afluentes el mes de septiembre presentó los valores más bajos de OD que oscilaron entre 3.0 a $4.0 \mathrm{mg} / \mathrm{L}$. Estos valores son similares y acordes a los valores de OD presentados en [32].

\section{Análisis del $\mathrm{pH}$}

En las seis campañas se pudo determinar que no hay una variación marcada en los valores de $\mathrm{pH}$ entre los puntos de muestreo (TABLA IV). En las primeras cuatro campañas se registraron valores de $\mathrm{pH}$ cercanas a 8.0 en todos los puntos de muestreo, a medida que se desciende en la profundidad de las aguas del embalse el pH disminuía cerca de 7.5, lo cual deja entrever la estratificación de sus aguas. Los valores básicos encontrados pueden deberse a diferentes explicaciones, una sería la alta tasa de descomposición de materia orgánica ubicada en la superficie del agua. Por otro lado, teniendo en cuenta los datos de precipitaciones del año 2017 del IDEAM [41] donde se muestra que en los meses de marzo a junio se presentó la época de mayor lluvia del año, es posible que de las partes altas de la cuenca del rio Magdalena se desprendieran minerales con una alta concentración de carbonatos, lo cual hace que se eleve el $\mathrm{pH}$ [32], [43]; otra posibilidad de la elevación del pH puede ser el consumo de $\mathrm{CO}_{2}$ en la fotosíntesis de plantas o algas acuáticas, lo cual eleva la concentración de carbonatos y bicarbonatos [43]. Se pudo comprobar en las dos últimas campañas que el $\mathrm{pH}$ disminuyó hacia valores neutros comprendidos entre 6.5 a 7.5, siendo el punto de muestreo Inicio del embalse el que presentó valores tendientes a ser ácidos con un mínimo de 6.0. Los afluentes presentaron el mismo comportamiento que los puntos en el embalse; los resultados obtenidos en el estudio realizado por EMGESA para el año 2014 a cada uno de los afluentes reportaron valores de $\mathrm{pH}$ entre 7.0 y 8.4 , siendo estas aguas ligeramente básicas [32].

\section{Análisis de la conductividad eléctrica (CE)}

En cuanto al comportamiento en las mediciones de CE dentro del embalse (Tabla IV) y analizando los puntos superficiales, conductividades que oscilaron entre los 90 y 110 $\mu \mathrm{S} / \mathrm{cm}$. Se puede interpretar que las aguas analizadas en los cuatro puntos superficiales del embalse presentan muy bajo contenido de salinidad, esto por su baja CE. En aguas naturales y superficiales una $\mathrm{CE}$ alrededor de $10 \mu \mathrm{S} / \mathrm{cm}$ puede indicar que predominan iones $\mathrm{Ca}^{+2}$ y $\mathrm{HCO}_{3}$, mientras que valores alrededor $110 \mu \mathrm{S} / \mathrm{cm}$ indica que predominan los iones $\mathrm{Na}^{+}$y $\mathrm{Ca}^{+2}$, razón por la cual hace pensar que la mayor concentración de sales disueltas puede deberse a carbonatos de sodio y de calcio, teniendo en cuenta los resultados obtenidos [44]. Sin embargo, no hay correlación entre el pH y la conductividad, esto debido a que se observa que la conductividad en los puntos del embalse casi se mantiene constante en las seis campañas, entre tanto el pH si varía, ligeramente básico en las primeras cuatro campañas y neutro en las dos últimas. De acuerdo con Castellanos et al. (2017) [32] la conductividad del río Magdalena varió entre 40 y 150 $\mu \mathrm{S} / \mathrm{cm}$, valores que concuerdan con los obtenidos en el presente estudio. En los afluentes se encontró que la conductividad fue ligeramente mayor. En la q. Garzón se registró un promedio de $106 \mu \mathrm{S} / \mathrm{cm}$, en la q. Guandinosa un promedio de $132 \mu \mathrm{S} / \mathrm{cm}$ y en el río Loro se registraron valores que fluctuaron entre los 58 y los $114 \mu \mathrm{S} / \mathrm{cm}$, teniendo un promedio durante los seis meses de muestreo de $95 \mu \mathrm{S} / \mathrm{cm}$. Los valores elevados de CE encontrados sobre los afluentes en el mes de mayo pudieron deberse a época de lluvias registrado por el IDEAM [37], ya que se puede producir aumento en escorrentías y trasladar desde las partes altas de la cuenca, sales minerales carbonáceas [43], [14].

\section{E. Análisis de la demanda bioquímica de oxígeno $\left(\mathrm{DBO}_{5}\right)$}

Los valores de $\mathrm{DBO}_{5}$ para los puntos superficiales dentro del embalse estuvieron por debajo de los $4 \mathrm{mg} / \mathrm{L}$ (Tabla III). En el estudio realizado por EMGESA se reporta que los niveles de $\mathrm{DBO}_{5}$ de las aguas de la cuenca alta del río Magdalena en la zona del embalse el Quimbo están por debajo de los $5 \mathrm{mg} / \mathrm{L}$ [32], valores similares a los encontrados en el presente estudio, con lo cual se puede establecer que las aguas del embalse tienen baja concentración de materia orgánica biodegradable. 
TABLA III

RESULTADOS DE LOS PARÁMETROS FISICOQUíMICOS DE LAS MUESTRAS DE AGUA TOMADAS EN EL EMBALSE EL QUIMBO.

\begin{tabular}{|c|c|c|c|c|c|c|c|c|c|c|c|c|c|c|c|c|c|c|c|c|c|c|}
\hline \multirow{2}{*}{\multicolumn{2}{|c|}{$\begin{array}{c}\text { Profundidad } \\
\text { Parámetro }\end{array}$}} & \multicolumn{7}{|c|}{$0.1 \mathrm{~m}$} & \multicolumn{7}{|c|}{$5 \mathrm{~m}$} & \multicolumn{7}{|c|}{$10 \mathrm{~m}$} \\
\hline & & \multirow[t]{2}{*}{$\mathrm{pH}$} & \multirow{2}{*}{$\begin{array}{c}\begin{array}{c}\text { Tem } \\
\mathbf{p}\end{array} \\
\left({ }^{\circ} \mathrm{C}\right) \\
\end{array}$} & \multirow{2}{*}{$\frac{\text { OD }}{(\mathrm{mg} / \mathrm{L})}$} & \multirow{2}{*}{$\begin{array}{c}\mathbf{C E} \\
\mu \mathrm{S} / \mathrm{c} \\
\mathrm{m}\end{array}$} & \multirow{2}{*}{$\begin{array}{c}\text { DBO } \\
(\mathrm{mg} / \mathrm{L})\end{array}$} & \multirow{2}{*}{$\begin{array}{c}\text { DQO } \\
(\mathrm{mg} / \mathrm{L}) \\
\end{array}$} & \multirow{2}{*}{$\begin{array}{c}\text { SST } \\
(\mathrm{mg} / \mathrm{L}) \\
\end{array}$} & \multirow[t]{2}{*}{ pH } & \multirow{2}{*}{$\begin{array}{c}\text { Tem } \\
\mathbf{p} \\
\left({ }^{\circ} \mathrm{C}\right)\end{array}$} & \multirow{2}{*}{$\frac{\text { OD }}{(\mathrm{mg} / \mathrm{L})}$} & \multirow{2}{*}{$\begin{array}{c}\mathbf{C E} \\
\mu \mathrm{S} / \mathrm{c} \\
\mathrm{m}\end{array}$} & & DQO & SST & pH & $\begin{array}{c}\text { Tem } \\
\text { p }\end{array}$ & OD & $\mathrm{CE}$ & Dво & DQO & $\begin{array}{l}\text { SS } \\
\text { T }\end{array}$ \\
\hline $\begin{array}{l}\text { Punto de } \\
\text { muestreo }\end{array}$ & Campaña & & & & & & & & & & & & $(\mathrm{mg} / \mathrm{L})$ & $(\mathrm{mg} / \mathrm{L})$ & $(\mathrm{mg} / \mathrm{L})$ & & $\left({ }^{\circ} \mathrm{C}\right)$ & $(\mathrm{mg} / \mathrm{L})$ & $\mu \mathrm{S} / \mathrm{cm}$ & $(\mathrm{mg} / \mathrm{L})$ & $(\mathrm{mg} / \mathrm{L})$ & $\begin{array}{c}(\mathrm{mg} \\
\mathrm{LL}) \\
\end{array}$ \\
\hline & 1 & 8,0 & 27,1 & 6,1 & 103,0 & $3.7 \pm 1.3$ & $20.5 \pm 7.8$ & 1,0 & 8 & 26,4 & 5,7 & 103,0 & $3.8 \pm 1.2$ & $17.5 \pm 4.9$ & 2,0 & 8,1 & 26,2 & 5,4 & 103,0 & $3.3 \pm 1.1$ & $21.0 \pm 2.8$ & 1,0 \\
\hline & 2 & 8,0 & 26,0 & 5,9 & 103,0 & $0.06 \pm 0.01$ & $17.5 \pm 3.6$ & 2,0 & 8,0 & 26,1 & 6,7 & 102,0 & $0.06 \pm 0.01$ & $16.5 \pm 4.9$ & 2,0 & 8,0 & 25,8 & 5,4 & 104,0 & $0.4 \pm 0.1$ & $27.0 \pm 5.7$ & 4,0 \\
\hline Compuerta & 3 & 8,3 & 24,9 & 5,8 & 102,0 & $2.4 \pm 0.9$ & $8.5 \pm 3.5$ & 2,0 & 8,3 & 24,8 & 5,8 & 102,0 & $3.0 \pm 1.1$ & $24.0 \pm 2.8$ & 2,0 & 8,3 & 25,2 & 5,8 & 101,0 & $2.4 \pm 0.8$ & $21.0 \pm 5.7$ & 2,0 \\
\hline s embalse & 4 & 8,7 & 29,6 & 5,7 & 103,0 & $0.6 \pm 0.2$ & $19.0 \pm 5.7$ & 1,7 & 8,6 & 27,2 & 5,6 & 104,0 & $0.9 \pm 0.3$ & $25.0 \pm 5.7$ & 2,0 & 8,7 & 25,8 & 5,4 & 118,0 & $0.6 \pm 0.2$ & $35 \pm 12$ & 0,4 \\
\hline & 5 & 7,4 & 25,9 & 4,5 & 102,0 & $0.5 \pm 0.2$ & $20.5 \pm 4.9$ & 4,0 & 7,4 & 25,6 & 4,3 & 103,0 & $0.6 \pm 0.2$ & $25.0 \pm 5.7$ & 2,0 & 7,5 & 25,5 & 4,4 & 104,0 & $0.4 \pm 0.1$ & $13.0 \pm 4.2$ & 4,0 \\
\hline & 6 & 6,8 & 27,0 & 4,7 & 102,0 & $0.8 \pm 0.3$ & $24.0 \pm 4.2$ & 2,3 & 6,5 & 26,1 & 4,5 & 102,0 & $0.9 \pm 0.4$ & $30.5 \pm 7.8$ & 1,7 & 5,8 & 26,2 & 4,4 & 103,0 & $0.9 \pm 0.3$ & $32.5 \pm 6.4$ & 2,3 \\
\hline & 1 & 8,1 & 23,8 & 6,9 & 169,0 & $5.3 \pm 2.1$ & $79.0 \pm 7.1$ & 220,0 & - & - & - & - & - & - & - & - & - & - & - & - & - & - \\
\hline & 2 & 8,1 & 22,3 & 6,7 & 102,0 & $2.7 \pm 0.5$ & $80.5 \pm 6.4$ & 388,0 & - & - & - & - & - & - & - & - & - & - & - & - & - & - \\
\hline Quebrada & 3 & 8,2 & 23,8 & 5,6 & 164,0 & $3.0 \pm 0.9$ & $40.0 \pm 5.7$ & 18,0 & - & - & - & - & - & - & - & - & - & - & - & - & - & - \\
\hline Guandinosa & 4 & 8,6 & 26,4 & 5,3 & 105,0 & $0.7 \pm 0.3$ & $31.0 \pm 9.9$ & 5,9 & - & - & - & - & - & - & - & - & - & - & - & - & - & - \\
\hline & 5 & 7,1 & 24,7 & 4,5 & 198,0 & $0.4 \pm 0.1$ & $9.5 \pm 2.1$ & 118,0 & - & - & - & - & - & - & - & - & - & - & - & - & - & - \\
\hline & 6 & 7,4 & 27,3 & 5,2 & 117,0 & $1.0 \pm 0.3$ & $26.5 \pm 10.6$ & 73,1 & - & - & - & - & - & - & - & - & - & - & - & - & - & - \\
\hline & 1 & 8,0 & 27,2 & 6,4 & 107,0 & $2.4 \pm 0.9$ & $17.5 \pm 4.9$ & 3,0 & 7,8 & 26,5 & 5,6 & 107,0 & $0.8 \pm 0.3$ & $29.0 \pm 11.3$ & 1,0 & 7,8 & 25,8 & 3,3 & 105,00 & $3.1 \pm 1.2$ & $46.0 \pm 9.9$ & 1,0 \\
\hline & 2 & 7,9 & 25,9 & 5,0 & 106,0 & $0.3 \pm 0.1$ & $24.0 \pm 1.4$ & 4,0 & 7,9 & 25,9 & 5,6 & 106,0 & $0.5 \pm 0.2$ & $25.0 \pm 9.9$ & 4,0 & 7,8 & 25,5 & 3,9 & 106,0 & $0.5 \pm 0.2$ & $41.0 \pm 12$ & 10, \\
\hline Gigante & 3 & 8,0 & 24,8 & 5,1 & 102,0 & $2.2 \pm 0.9$ & $41.5 \pm 0.7$ & 5,0 & 8,0 & 24,9 & 5,2 & 102,0 & $2.4 \pm 0.9$ & $57.0 \pm 0.0$ & 3,0 & 7,9 & 24,9 & 5,0 & 104,0 & $2.6 \pm 1.0$ & $41.0 \pm 8.5$ & 4,0 \\
\hline & 4 & 8,7 & 27,7 & 5,7 & 101,0 & $1.0 \pm 0.4$ & $10 \pm 2.4$ & 3,8 & 8,5 & 26,4 & 5,8 & 101,0 & $1.1 \pm 0.4$ & $15.5 \pm 0.7$ & 2,1 & 8,3 & 25,3 & 4,7 & 108,0 & $0.3 \pm 0.1$ & $25-0 \pm 4.2$ & 1,8 \\
\hline & 5 & 7,3 & 26,3 & 4,2 & 102,0 & $0.5 \pm 0.2$ & $36.0 \pm 4.2$ & 4,0 & 7,2 & 25,7 & 4,1 & 103,0 & $0.8 \pm 0.3$ & $18.5 \pm 0.7$ & 5,0 & 7,2 & 25,7 & 4,0 & 102,0 & $0.7 \pm 0.2$ & $13.5 \pm 4.9$ & 2,0 \\
\hline & 6 & 7,4 & 26,8 & 4,8 & 103,0 & $0.9 \pm 0.3$ & $33.0 \pm 5.7$ & 3,2 & 7,3 & 26,4 & 4,6 & 103,0 & $0.9 \pm 0.4$ & $19.5 \pm 3.5$ & 2,0 & 7,3 & 26,0 & 4,0 & 103,0 & $1.0 \pm 0.4$ & $28.0 \pm 1.4$ & 3,6 \\
\hline & 1 & 8,1 & 23,0 & 6,8 & 107,0 & $3.1 \pm 1.1$ & $52.0 \pm 11.3$ & 63,0 & - & - & - & - & - & - & - & - & - & - & - & - & - & - \\
\hline & 2 & 8,2 & 19,2 & 7,1 & 58,0 & $2.2 \pm 0.5$ & $70.5 \pm 9.2$ & 264,0 & - & - & - & - & - & - & - & - & - & - & - & - & - & - \\
\hline & 3 & 8,2 & 21, & 5,8 & 101,0 & $3.4 \pm 1.3$ & $31.5 \pm 9.2$ & 11,0 & - & - & - & - & - & - & - & - & - & - & - & - & - & - \\
\hline Río Loro & 4 & 8,7 & 23,8 & 5,9 & 107,0 & $0.8 \pm 0.2$ & $38.5 \pm 9.2$ & 31,4 & - & - & - & - & - & - & - & - & - & - & - & - & - & - \\
\hline & 5 & 7,2 & 21,4 & 4,5 & 114,0 & $0.8 \pm 0.2$ & $38.5 \pm 10.6$ & 14,0 & - & - & - & - & - & - & - & - & - & - & - & - & - & - \\
\hline & 6 & 7,1 & 22,3 & 5,0 & 84,0 & $0.8 \pm 0.3$ & $27.5 \pm 6.4$ & 15,8 & & - & - & - & - & - & - & - & - & - & - & - & - & - \\
\hline & 1 & 8,0 & 27,8 & 6,5 & 109,0 & $2.1 \pm 0.8$ & $33.0 \pm 8.5$ & 1,0 & 7,7 & 26,4 & 5,3 & 109,0 & $0.9 \pm 0.4$ & $40.0 \pm 16$ & 2,0 & 7,8 & 25,4 & 2,6 & 108,0 & $1.4 \pm 0.2$ & $49.0 \pm 18.4$ & 3,0 \\
\hline & 2 & 8,1 & 26,4 & 6,2 & 109,0 & $0.20 \pm 0.01$ & $15.0 \pm 4.2$ & 3,0 & 7,9 & 26,2 & 5,7 & 108,0 & $0.12 \pm 0.01$ & $31.5 \pm 0.7$ & 2,0 & 7,5 & 25,0 & 3,2 & 103,0 & $0.14 \pm 0.02$ & $18.5 \pm 0.7$ & 3,0 \\
\hline & 3 & 8,0 & 24,6 & 5,1 & 102,0 & $3.1 \pm 0.5$ & $20.0 \pm 4.2$ & 3,0 & 7,9 & 24,5 & 5,0 & 102,0 & $3.1 \pm 1.2$ & $21.5 \pm 4.9$ & 3,0 & 8,0 & 24,6 & 5,0 & 102,0 & $3.6 \pm 1.2$ & $32.5 \pm 7.8$ & 4,0 \\
\hline La Trina & 4 & 8,9 & 27,8 & 6,7 & 102,0 & $1.3 \pm 0.4$ & $42.0 \pm 1.4$ & 2,5 & 8,5 & 27,0 & 5,8 & 101,0 & $0.9 \pm 0.2$ & $30 \pm 4.2$ & 1,0 & 7,9 & 25,1 & 3,5 & 106,0 & $0.8 \pm 0.2$ & $20.0 \pm 5.7$ & 2,1 \\
\hline & 5 & 7,3 & 26,4 & 4,4 & 103,0 & $0.8 \pm 0.3$ & $28.0 \pm 4.2$ & 4,0 & 6,8 & 25,9 & 4,0 & 102,0 & $0.5 \pm 0.2$ & $37.5 \pm 3.5$ & 1,0 & 6,5 & 25,0 & 2,5 & 102,0 & $0.5 \pm 0.2$ & $50.5 \pm 3.5$ & 2,0 \\
\hline & 6 & 7,0 & 27,9 & 4,4 & 103,0 & $1.0 \pm 0.4$ & $18.0 \pm 4.2$ & 2,9 & 6,8 & 26,6 & 4,5 & $\begin{array}{l}102,0 \\
102,0\end{array}$ & $1.0 \pm 0.3$ & $16.5 \pm 3.5$ & 2,7 & 6,6 & 24,8 & 1,9 & 96,0 & $0.7 \pm 0.3$ & $24.5 \pm 6.4$ & 0,8 \\
\hline & 1 & 8,3 & 26,9 & 7,0 & 114,0 & $3.4 \pm 1.1$ & $40.0 \pm 4.2$ & 1,0 & 8,6 & 23,2 & 7,7 & 85,0 & $2.7 \pm 1.1$ & $44.5 \pm 4.95$ & 9,0 & 7,9 & 21,6 & 6,4 & 74,00 & $2.6 \pm 0.7$ & $54.0 \pm 8.5$ & $\begin{array}{c}24, \\
0\end{array}$ \\
\hline & 2 & 8,0 & 25,5 & 6,3 & 111,0 & $0.2 \pm 0.1$ & $33.5 \pm 2.1$ & 6,5 & 7,6 & 21,7 & 6,1 & 80,0 & $1.0 \pm 0,3$ & $27.0 \pm 7.1$ & 82,0 & 7,3 & 21,2 & 6,6 & 88,0 & $1.9 \pm 0.5$ & $29.5 \pm 4.9$ & $\begin{array}{c}115 \\
0\end{array}$ \\
\hline Inicio & 3 & 7,8 & 23,8 & 4,9 & 104,0 & $2.7 \pm 0.7$ & $32.5 \pm 2.1$ & 11,0 & 7,7 & 22,6 & 4,8 & 85,0 & $2.8 \pm 1.0$ & $29.5 \pm 4.9$ & 28,0 & 7,7 & 21,4 & 4,7 & 80,0 & $3.7 \pm 1.4$ & $56.5 \pm 13.4$ & 27, \\
\hline & 4 & 7,9 & 26,8 & 5,7 & 100,0 & $0.6 \pm 0.2$ & $16.0 \pm 2.8$ & 4,7 & 7,4 & 25,0 & 4,7 & 98,0 & $0.8 \pm 0.3$ & $15.0 \pm 4.2$ & 9,6 & 6,8 & 24,1 & 4,6 & 140,0 & $1.2 \pm 0.4$ & $6.0 \pm 1.4$ & 3,3 \\
\hline & 5 & 6,0 & 24,9 & 3,7 & 101,0 & $0.4 \pm 0.1$ & $6.0 \pm 1.4$ & 4,0 & 5,7 & 24,1 & 3,7 & 95,0 & $0.6 \pm 0.2$ & $35.0 \pm 14.1$ & 5,0 & 5,0 & 22,5 & 4,0 & 120,0 & $1.6 \pm 0.2$ & $20.5 \pm 7.8$ & 9,0 \\
\hline & 6 & 6,1 & 26,5 & 4,3 & 102,0 & $0.5 \pm 0.2$ & $13.5 \pm 3.5$ & 3,1 & 5,6 & 24,6 & 4,3 & 93,0 & $0.9 \pm 0.35$ & $23.5 \pm 0.7$ & 5,6 & 5,1 & 22,4 & 4,2 & 87,0 & $1.0 \pm 0.25$ & $28.5 \pm 3.5$ & $\begin{array}{l}16, \\
8\end{array}$ \\
\hline & 1 & 8,0 & 25,0 & 5,6 & 126,0 & $8.5 \pm 3.4$ & $73.0 \pm 5.7$ & 15,0 & - & - & - & - & - & 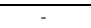 & - & - & - & - & - & - & - & - \\
\hline & 0 & 8, & 19,9 & 6,3 & 103,0 & $5.2 \pm 1$ & & 122,0 & - & - & 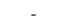 & - & - & 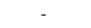 & - & - & 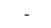 & - & - & - & - & - \\
\hline Quebrada & 3 & 8, & 24 & 5,6 & 96 & 1.2 & & 16 & - & - & - & - & - & - & - & - & - & - & - & - & - & - \\
\hline Garzón & 4 & 8,0 & 25 , & 4,2 & 104,0 & $1.0 \pm 0.2$ & 27 & 5, & - & - & - & - & - & - & - & - & - & - & - & - & - & - \\
\hline & 5 & 6,3 & 24,7 & 3,3 & 106,0 & $0.6 \pm 0.2$ & $19.5 \pm 3.5$ & 5,0 & - & - & - & - & - & - & - & - & - & - & - & - & - & - \\
\hline & 6 & 6,5 & 26,7 & 4,5 & 101,0 & $1.0 \pm 0.35$ & $30.0 \pm 7.1$ & 2,7 & - & - & - & - & - & - & - & - & - & - & - & - & - & - \\
\hline
\end{tabular}

En la Tabla IV se puede observar una clasificación del agua según el contenido de $\mathrm{DBO}_{5}$ propuesto en [45], donde se puede concluir que las aguas del embalse no están contaminadas por desechos orgánicos biodegradables antropogénicos. La q. Garzón presentó valores de $\mathrm{DBO}_{5}$ aproximadamente de $10 \mathrm{mg} / \mathrm{L}$ en los meses de mayo y junio. No obstante, no se observa relación alguna con la elevación de los SST; la contaminación puede ser por sólidos disueltos. Teniendo en cuenta la Tabla IV [45], las aguas del afluente pudieron estar levemente contaminadas por material biodegradable disuelto, proveniente de aguas residuales domésticas. La q. Guandinosa y el r. Loro registraron valores por debajo de 7 y $4 \mathrm{mg} / \mathrm{L}$ respectivamente.

Las elevaciones en los valores de $\mathrm{DBO}_{5}$ observadas se dieron durante los meses de mayores precipitaciones, en relación con lo expuesto por el IDEAM [41]. Analizando los SST en los dos primeros meses en la q. Guandinosa vemos que aumentaron considerablemente con respecto a los demás meses, de tal forma que estos sólidos pueden deberse a sustancias no biodegradables que provienen de material desprendido y arrastrado por las escorrentías [32]. En general, en todos los puntos de muestreo en los demás meses, temporada de menores precipitaciones, la $\mathrm{DBO}_{5}$ estuvo por debajo de los $2 \mathrm{mg} / \mathrm{L}$ indicando que estas aguas no presentaron una evidente contaminación por materia biodegradable [45].
TABLA IV

CLASIFICACIÓN DEL AGUA TENIENDO EN CUENTA LA DEMANDA BIOQUÍMICA DE OXÍGENO $\left(\mathrm{DBO}_{5}\right)$ [45].

\begin{tabular}{|c|c|c|}
\hline Valor de DBO5 & Criterio & Descripción \\
\hline $\mathrm{DBO} \leq 3 \mathrm{ppm}$ & Excelente & No contaminada \\
\hline $3 \mathrm{ppm}<\mathrm{DBO} \leq 6 \mathrm{ppm}$ & Buena calidad & $\begin{array}{c}\text { Aguas superficiales con bajo } \\
\text { contenido de materia orgánica } \\
\text { biodegradable. }\end{array}$ \\
\hline $6 \mathrm{ppm}<\mathrm{DBO} \leq 30 \mathrm{ppm}$ & Aceptable & $\begin{array}{c}\text { Con indicio de contaminación. } \\
\text { Aguas superficiales con } \\
\text { capacidad de autodepuración o } \\
\text { descargas de aguas residuales } \\
\text { tratadas biológicamente. }\end{array}$ \\
\hline $30 \mathrm{ppm}<\mathrm{DBO} \leq 120$ & Contaminada & $\begin{array}{c}\text { Aguas superficiales con } \\
\text { descargas de aguas residuales } \\
\text { crudas, principalmente de origen } \\
\text { municipal. }\end{array}$ \\
\hline $\mathrm{pBm}>120 \mathrm{ppm}$ & $\begin{array}{c}\text { Fuertemente } \\
\text { contaminada }\end{array}$ & $\begin{array}{c}\text { Aguas superficiales con fuerte } \\
\text { impacto de descargas de aguas } \\
\text { residuales crudas municipales y } \\
\text { no municipales. }\end{array}$ \\
\hline
\end{tabular}

\section{F. Análisis de la demanda química de oxígeno (DQO)}

En los puntos superficiales dentro del embalse la DQO estuvo por debajo de $42 \mathrm{mg} / \mathrm{L}$ (Tabla III), lo que se puede interpretar como un agua aceptable al contrastar con la información consignada en la Tabla V [45]. Por lo tanto, se considera que la mayoría de los puntos se encuentran en un nivel óptimo [45]. El punto más alto se registró en el mes de agosto, esto debido a la precipitación registrada en la estación de Garzón, la cual presentó un índice de precipitación del $145 \%$ [41]. Además, en la estación de Pitalito en el mes de Julio se presentó una precipitación del $125 \%$ y en agosto fue 
$>170 \%$. Generando así un arrastre en materia orgánica proveniente del rio Magdalena la cual pudo provocar un incremento de DQO en este mes. En los tres afluentes que convergen en el embalse, la DQO presentó un promedio de 40 $\mathrm{mg} / \mathrm{L}$, por lo tanto, son consideradas como aguas óptimas [45]. Sin embargo, en la segunda salida de campo llevada a cabo en el mes de junio la DQO aumentó; en la q. Garzón se presentó un registro de $79 \mathrm{mg} / \mathrm{L}$, en el r. Loro una concentración de $70.5 \mathrm{mg} / \mathrm{L}$, y en la q. Guandinosa, para ese mismo mes, se obtuvo un valor de $80 \mathrm{mg} / \mathrm{L}$, esto debido a bajas precipitaciones y elevadas temperaturas durante el mes [41], esto produjo una alta acumulación de materia orgánica en descomposición, la cual fue arrastrada durante los meses de precipitaciones elevadas [32]. Las aguas de los afluentes, como se ha descrito anteriormente, presentan contaminación leve debida a material orgánico proveniente de aguas residuales de los municipios por donde cruzan estos cuerpos de agua.

TABLA V

CLASIFICACIÓN DEL AGUA TENIENDO EN CUENTA LA DEMANDA QUíMICA DE OXÍGENO (DQO) [45].

\begin{tabular}{|c|c|c|}
\hline Valor de DQO & Criterio & Descripción \\
\hline $\mathrm{DBO} \leq 10 \mathrm{ppm}$ & Excelente & No contaminada \\
\hline $10 \mathrm{ppm}<\mathrm{DQO} \leq 20 \mathrm{ppm}$ & Buena calidad & $\begin{array}{c}\text { Aguas superficiales con bajo } \\
\text { contenido de materia orgánica } \\
\text { biodegradable. }\end{array}$ \\
\hline $20 \mathrm{ppm}<\mathrm{DBO} \leq 40 \mathrm{ppm}$ & Aceptable & $\begin{array}{c}\text { Con indicio de contaminación. } \\
\text { Aguas superficiales con } \\
\text { capacidad de autodepuración o } \\
\text { descargas de aguas residuales } \\
\text { tratadas biológicamente. }\end{array}$ \\
\hline $40 \mathrm{ppm}<\mathrm{DBO} \leq 200$ & Contaminada & $\begin{array}{c}\text { Aguas superficiales con } \\
\text { descargas de aguas residuales } \\
\text { crudas, principalmente de origen } \\
\text { municipal. }\end{array}$ \\
\hline $\mathrm{DBO}>200 \mathrm{ppm}$ & Fuertemente & $\begin{array}{c}\text { Aguas superficiales con fuerte } \\
\text { impacto de descargas de aguas } \\
\text { residuales crudas municipales y } \\
\text { no municipales. }\end{array}$ \\
\hline
\end{tabular}

\section{G. Análisis de los sólidos suspendidos totales (SST)}

Los resultados con respecto a los sólidos suspendidos totales en aguas superficiales, dentro del embalse oscilan entre 1 y $11 \mathrm{mg} / \mathrm{L}$ (Tabla III). El mes de mayo registró el valor más bajo de SST, lo cual es congruente con lo que se había descrito con anterioridad, ya que en mayo hubo un aumento en las precipitaciones [32]. No obstante, el mes de julio registró el valor más elevado, ya que en el mes de julio hubo bajas precipitaciones y elevadas temperaturas, produciendo una mayor acumulación de sólidos no disueltos en estas aguas. Estos resultados expuestos indican que la calidad del agua para los puntos dentro del embalse es excelente, debido a que presenta poca presencia de sólidos suspendidos. La q. Guandinosa fue el afluente con mayor promedio de SST (136 $\mathrm{mg} / \mathrm{L}$ ), mientras que la q. Garzón es el afluente con menor valor de SST. Sin embargo, se consideran aguas con bajo nivel de SST correspondiente con lo encontrado en el estudio realizado por EMGESA [32].

\section{H. Análisis de la calidad del agua ISQA}

Los datos obtenidos al aplicar la ecuación (1) de la clasificación de ISQA indicaron que en promedio general el agua del embalse el Quimbo se encuentra en el rango de 60-80 unidades ISQA, por lo cual se clasifica como de buena calidad (Tabla I). De acuerdo con esto, estas aguas se las puede usar para consumo humano con tratamientos convencionales, recreación, riego y uso industrial [31]. Si bien la clasificación está mostrando que el agua del embalse el Quimbo es buena y en principio puede tener diversos usos, el estudio muestra que hay zonas de especial cuidado, donde se debe mitigar el impacto ambiental negativo producto de la descomposición de material vegetal que fue talado cuando se empezó a adecuar el terreno para el llenado del embalse, del crecimiento de plantas acuáticas, del aumento de la sedimentación y a su vez del aumento los sólidos que están disueltos o suspendidos en las aguas, esto con el objetivo de que el agua mantenga en el tiempo su buena calidad incluso en periodos de escasas o elevadas precipitaciones y aumentos de temperatura.

Un análisis más detallado de cada uno de los puntos de muestreo, empezando por el punto donde comienza el embalse hasta donde termina, se muestra a continuación. En el inicio del embalse se encontró que en los meses de mayo y junio las aguas a $10 \mathrm{~m}$ de profundidad presentaron valores ISQA menores que 60 unidades pudiendo clasificarlas como de mediana calidad (Tabla VI). Estos valores obtenidos se dieron en temporada de mayor precipitación [41], razón por la cual estas aguas tuviesen una carga elevada de sedimentos que hicieran alterar su clasificación como de buena calidad, además teniendo en cuenta que en el punto de muestreo llegan directamente las aguas del río Magdalena. El siguiente punto de muestreo aguas abajo en el embalse fue el denominado la Trina, punto en el cual se determinó calidad media del agua en los meses de mayo y septiembre (Tabla VI) a 5 y $10 \mathrm{~m} \mathrm{de}$ profundidad. Estos resultados pudieron ser causados por los altos registros de DQO por acumulación de materia orgánica debida a tiempo seco en el mes de septiembre y una baja concentración de OD, lo que hizo que su calidad disminuyera. Mientras que, en los meses de junio, julio, agosto, octubre se registró una calidad buena. Continuando aguas abajo en el embalse se encuentra el punto denominado Gigante acostado, quizás el punto de muestreo dentro del embalse con mayores alteraciones. Este punto presentó en los meses de mayo (a 10 $\mathrm{m}$ ), junio (a $10 \mathrm{~m}$ ), julio (a $5 \mathrm{~m}$ ) y septiembre (superficial a 0.1 m) una clasificación ISQA de calidad mediana (Tabla VI). Estos cambios en la calidad del agua pudo ser por aumentos en la $\mathrm{DBO}_{5}$, la $\mathrm{DQO}$ y una baja significativa de la concentración de OD, indicándonos una alta concentración de materia orgánica en descomposición que posibilita el aumento y crecimiento de bacterias nativas que alteran el ecosistema consumiendo gran cantidad de oxígeno. 
TABLA VI

RESULTADOS DE LA CLASIFICACIÓN ISQA DEL AGUA DEL EMBALSE EL QUIMBO.

\begin{tabular}{|c|c|c|c|c|c|c|c|}
\hline \multicolumn{2}{|c|}{ Profundidad } & \multicolumn{2}{|c|}{$0.1 \mathrm{~m}$} & \multicolumn{2}{|c|}{$5 \mathrm{~m}$} & \multicolumn{2}{|c|}{$10 \mathrm{~m}$} \\
\hline $\begin{array}{l}\text { Punto de } \\
\text { muestreo }\end{array}$ & Campaña & $\begin{array}{c}\text { Clasificación } \\
\text { ISQA }\end{array}$ & Calidad agua & $\begin{array}{l}\text { Clasificación } \\
\text { ISQA }\end{array}$ & Calidad agua & $\begin{array}{c}\text { Clasificación } \\
\text { ISQA }\end{array}$ & Calidad agua \\
\hline \multirow{7}{*}{$\begin{array}{l}\text { Compuertas } \\
\text { embalse }\end{array}$} & 1 & 68,2 & Buena & 68,9 & Buena & 67,3 & Buena \\
\hline & 2 & 74,1 & Buena & 69,9 & Buena & 65,4 & Buena \\
\hline & 3 & 76,6 & Buena & 68,4 & Buena & 70,2 & Buena \\
\hline & 4 & 65,5 & Buena & 64,9 & Buena & 62,4 & Buena \\
\hline & 5 & 65,5 & Buena & 63,9 & Buena & 67,8 & Buena \\
\hline & 6 & 64,0 & Buena & 62,3 & Buena & 61,7 & Buena \\
\hline & PROM & 69,0 & Buena & 66,4 & Buena & 65,8 & Buena \\
\hline \multirow{7}{*}{$\begin{array}{c}\text { Quebrada } \\
\text { Guandinosa }\end{array}$} & 1 & 35,2 & Mala & - & - & - & - \\
\hline & 2 & 36,9 & Mala & - & - & - & - \\
\hline & 3 & 58,9 & Mediana & - & - & - & - \\
\hline & 4 & 63,0 & Buena & - & - & - & - \\
\hline & 5 & 54,4 & Mediana & - & - & - & - \\
\hline & 6 & 51,7 & Mediana & - & - & - & - \\
\hline & PROM & 50,0 & Mediana & - & - & - & - \\
\hline \multirow{7}{*}{$\begin{array}{l}\text { Gigante } \\
\text { acostado }\end{array}$} & 1 & 69,3 & Buena & 64,8 & Buena & 54,7 & Mediana \\
\hline & 2 & 66,5 & Buena & 64,8 & Buena & 56,5 & Mediana \\
\hline & 3 & 60,9 & Buena & 55,9 & Mediana & 60,9 & Buena \\
\hline & 4 & 72,2 & Buena & 69,8 & Buena & 64,9 & Buena \\
\hline & 5 & 59,2 & Mediana & 65,0 & Buena & 67,8 & Buena \\
\hline & 6 & 61,3 & Buena & 65,7 & Buena & 61,8 & Buena \\
\hline & PROM & 64,9 & Buena & 64,3 & Buena & 61,1 & Buena \\
\hline \multirow{7}{*}{ Río Loro } & 1 & 54,0 & Mediana & - & - & - & - \\
\hline & 2 & 42,1 & Mala & - & - & - & - \\
\hline & 3 & 67,4 & Buena & - & - & - & - \\
\hline & 4 & 60,9 & Buena & - & - & - & - \\
\hline & 5 & 61,0 & Buena & - & - & - & - \\
\hline & 6 & 66,7 & Buena & - & - & - & - \\
\hline & PROM & 58,7 & Mediana & - & - & - & - \\
\hline \multirow{7}{*}{ La Trina } & 1 & 63,3 & Buena & 60,3 & Buena & 52,0 & Mediana \\
\hline & 2 & 71,6 & Buena & 65,2 & Buena & 63,8 & Buena \\
\hline & 3 & 68,4 & Buena & 68,4 & Buena & 63,8 & Buena \\
\hline & 4 & 62,0 & Buena & 64,7 & Buena & 64,0 & Buena \\
\hline & 5 & 62,1 & Buena & 59,2 & Mediana & 51,9 & Mediana \\
\hline & 6 & 64,1 & Buena & 66,2 & Buena & 59,3 & Mediana \\
\hline & PROM & 65,3 & Buena & 64,0 & Buena & 59,1 & Mediana \\
\hline \multirow{7}{*}{$\begin{array}{c}\text { Inicio } \\
\text { embalse }\end{array}$} & 1 & 63,8 & Buena & 67,6 & Buena & 58,7 & Mediana \\
\hline & 2 & 65,5 & Buena & 61,2 & Buena & 55,9 & Mediana \\
\hline & 3 & 62,5 & Buena & 63,5 & Buena & 57,0 & Buena \\
\hline & 4 & 68,9 & Buena & 67,8 & Buena & 67,9 & Buena \\
\hline & 5 & 74,0 & Buena & 60,6 & Buena & 63,3 & Buena \\
\hline & 6 & 66,7 & Buena & 65,2 & Buena & 64,3 & Buena \\
\hline & PROM & 66,9 & Buena & 64,3 & Buena & 61,2 & Buena \\
\hline \multirow{7}{*}{$\begin{array}{c}\text { Quebrada } \\
\text { Garzón }\end{array}$} & 1 & 54,4 & Mediana & - & - & - & - \\
\hline & 2 & 45,3 & Mediana & - & - & - & - \\
\hline & 3 & 60,1 & Buena & - & - & - & - \\
\hline & 4 & 57,8 & Mediana & - & - & - & - \\
\hline & 5 & 63,6 & Buena & - & - & - & - \\
\hline & 6 & 61,9 & Buena & - & - & - & - \\
\hline & PROM & 57,2 & Mediana & - & - & - & - \\
\hline
\end{tabular}


El último punto de muestreo ubicado en las compuertas del embalse el Quimbo mantuvo durante los seis meses de muestreo una clasificación ISQA de buena calidad (Tabla VII). A nivel general en este punto se obtuvo un promedio de 67 unidades ISQA siendo el más alto registrado entre los siete puntos de muestreo. En cuanto a los afluentes que ayudan a surtir de agua al embalse, aparte del río Magdalena como principal fuente de suministro, la q. Garzón, el río Loro y la q. Guandinosa el estudio mostró en promedio que sus aguas presentan calidad mediana de acuerdo con la clasificación ISQA (Tabla VII). Sin embargo, se pudo observar que la q. Guandinosa fue el afluente ambientalmente más afectado, puesto que en los dos primeros meses de muestreo las aguas presentaron una mala calidad según ISQA; en el tercer, quinto $\mathrm{y}$ sexto mes estas aguas se clasificaron como de mediana calidad. Estos resultados se obtuvieron porque se encontraron valores elevados en los SST y en la DQO.

El estudio llevado a cabo por [32] se encontró que el curso de este cuerpo de agua está alterado, lo anterior debido al dragado que se ha realizado para evitar posibles inundaciones en las tierras aledañas, observándose gran cantidad de material rocoso hacia las orillas de la quebrada. También el estudio [32] indica que hay afectación sobre el bosque nativo que crece en los alrededores del cuerpo de agua cerca de la desembocadura a la represa, donde se encontró árboles sembrados por las comunidades aledañas y lugares donde predominan plantas herbáceas. Estos problemas sobre la quebrada hacen que se disminuya la posibilidad de filtración y retención de sólidos por parte de plantas silvestres propias de la cuenca hidrográfica. La calidad del agua de la q. Garzón según la clasificación del ISQA (Tabla VI), para los meses de mayo, junio y agosto fue de mediana calidad, esto se debió valores elevados en la DQO y los SST, ocasionando la disminución en la calidad. Mientras que en los meses de julio, septiembre y octubre se registró como de buena calidad, lo cual indica una mejora en las características del líquido. Esta quebrada pasa por el casco urbano del municipio de Garzón, recibiendo los vertimientos de las aguas residuales municipales.

En el estudio llevado a cabo en [32], se mostró evidencia de que las laderas del cauce del río presentan erosión en varios de sus tramos, así como también vegetación introducida por el hombre a modo de jardinería. Con respecto al río Loro el estado de la calidad del agua con relación a la tabla de clasificación del ISQA (Tabla VI), mostró que los meses de mayo y junio el estado del agua fue de mediana calidad, esto causado por el aumento en los SST y DQO. En cambio, en los meses de julio, agosto, septiembre y octubre se logró calificarla como de buena calidad, indicando que existió una mejora en sus características. En [32] se pudo evidenciar que en algunos sectores del cauce del río se encuentra obstruido con sacos de arena y material rocoso para fines turísticos, así mismo, se encontró proyectos piscícolas que hacen uso de sus aguas y bosque intervenido para la implementación de estos proyectos económicos. No obstante, este cuerpo de agua es el que mejor salud presenta con respecto a los otros afluentes que hicieron parte del presente estudio. A pesar de las evidentes afectaciones en cada uno de los afluentes, sus aguas mantienen un promedio de calidad mediana, de tal manera que estas aguas pueden ser utilizadas para actividades como riego, uso industrial, recreación y consumo humano con tratamientos especiales [31].

\section{CONCLUSIONES}

Utilizando el Índice Simplificado de Calidad de Agua (ISQA), se logró establecer el estado en que se encuentra la masa de agua del embalse El Quimbo; los puntos de muestreo ubicados en el área de inundación evidenciaron valores desde 51.9 hasta 76.6, rango en el cual se puede clasificar como aguas de buena calidad, con potencial uso en pesca, uso recreativo, además de fuente para acueductos con sistemas de tratamientos convencionales [31].

Los análisis realizados durante el tiempo de investigación permitieron diagnosticar la calidad de agua de los principales afluentes que desembocan al embalse El Quimbo (Quebrada Garzón, río Loro y la quebrada Guandinosa). Los resultados obtenidos en el ISQA se encuentran entre 35.2 y 67.4, estos tres afluentes presentaron una mediana calidad en el agua, esta clasificación está relacionada principalmente a los altos valores de la materia orgánica, evidenciados en los resultados de la DQO, en donde se pudieron observar los niveles más altos obtenidos durante la investigación.

\section{AGRADECIMIENTOS}

Los autores reconocen las contribuciones de O. A. Aguilera, J. N. Castro en la obtención de los datos experimentales de este documento. Agradecen a N. H. Ortíz la ayuda en la elaboración del mapa de la ubicación geográfica del Quimbo.

\section{REFERENCIAS}

[1] Brauman, K. A., Daily, G. C., Duarte T. K., y Mooney, H. A. "The nature and value of ecosystem services: An overview highlighting hydrologic services," Annual Review of Environment and Resources, vol. 32, pp. 67-98, 2007.

[2] Ríos, M. L. y Torres, D. "Determinación de metales pesados por métodos analíticos en residuos de sulfato de zinc producidos en un laboratorio de química," Entre Ciencia e Ingeniería, vol. 3, n 5 , pp. 9-29, 2009.

[3] P. Torres, P., Cruz, C. H. y Patiño, P. J. "Índices de calidad de agua en fuentes supeficiales utilizadas en la producción de agua para consumo humano. Una revisión crítica," Revista de ingenierias Universidad de Medellín, vol. 8, n 15, pp. 79-94, 2009.

[4] Carvajal, Y. "Efectos de la variabilidad climática (vc) y el cambio climático (cc) en los recursos hídricos de Colombia," Entre Ciencia e Ingeniería, vol. 5, nº 9, pp. 33-61, 2011.

[5] Meléndez, K. J. y Losada, L. C. "Cálculo de la ingesta diaria de flúor en el agua potable, determinada a partir de la ingesta de agua calculada del centro poblado el Juncal, municipio de Palermo, departamento del Huila", de Investigación formativa en ingeniería, Medellín, Colombia: Editorial Instituto Antioqueño de Investigación, 2017. (1st ed). [Online]. Disponible: https://doi.org/10.5281/zenodo.2613950

[6] González, J. S., Carvajal, L. A. y Cuadro, O. F. Estado del índice de escasez de agua superficial en el departamento del Huila, de Saberes emergentes para la cuarta revolución industrial, Neiva, Colombia: Editorial Corhuila, 2019. (1st ed).

[7] Nilson, C., Reidy, C. A., Dynesius, M. y Revenga, C. "Fragmentation and flow regulation of the world's large rivers systems," Science, vol. 308 , pp. 405-408, 2005 
[8] Finer, M. y Jenkins, C. N. "Proliferation of hydroelectric dams in the Andean Amazon and implications for Andes-Amazon connectivity," PLoS ONE, vol. 7, nº 4, pp. e35126, 2012.

[9] Anderson, E., Freeman, M. y Pringle, C. "Ecological consequences of hydropower development in Central America: Impacts of small dams and water diversion on neotropical stream fish assemblages," River Research and applications, vol. 22, pp. 397-411, 2006.

[10] Gehrke, P.C., Gilligan, D. M. y Barwick, M. "Changes in fish communities of the shoalhaven river 20 years after construction of Tallowa dam, Australia," River research and applications, vol. 18, pp. 265-286, 2002.

[11] Penczak, T. y Kruk, A. "Threatened obligatory riverine fishes in human-modified Polish rivers," Ecology of Freshwater Fish, vol. 9, pp. 109-117, 2000.

[12] Darwish, M. A. G. "Geochemistry of the High Dam Lake sediments, south Egypt: implications for environmental significance," International Journal of Sediment Research, vol. 28, $\mathrm{n}^{\circ}$ 4, pp. 544-559, 2013.

[13] Fonseca, R., Pinho, C. y Oliveira, M. "The influence of particles recycling on the geochemistry of sediments in a large tropical dam lake in the Amazonian region, Brazil," Journal of South American Earth Sciences, vol. 72, pp. 328-350, 2016.

[14] Cifuentes, G. R., Gamboa R. A. y Rocha, Z. E. "Diagnóstico fisicoquímico, biológico y microbiológico de las aguas del embalse de la copa (Boyacá), Tunja, Boyacá”: Ediciones Universidad de Boyacá, 2014.

[15] Bunea, F., Bucur, D. M., Dumitran G. E. y Ciocan, G. D. "Water Quality in Hydroelectric Sites," de Ecological Water Quality: Water Treatment and Reuse, Rijeka, Croatia, IntechOpen, 2012. (1st ed).

[16] Gutiérrez, L. R., Chávez, L. M. y Arregui, L. "Physico-chemical and microbiological analysis of water of the Presa de los Patos in the Desierto de los leones National Park, Mexico," Advances in Biological Chemistry, vol. 7, pp. 122-138, 2017.

[17] Castro, M., Almaida, J., Ferrer, J. y Díaz, D. "Indicadores de la calidad del agua: evolución y tendencias a nivel global," Ingeniería Solidaria, vol. 10, no 17, pp. 111-124, 2014

[18] Rangeti, I., Dzwairo, B., Barratt, G. y Otieno, F. "Ecosystem-specific water quality indices," African Journal of Aquatic Science, vol. 40, n 3, pp. 227-234, 2015.

[19] Jurado, M. A. y Mercado, I. D "Revisión sistemática de técnicas no convencionales para la evaluación de la calidad del agua de ríos contaminados con plaguicidas," Entre Ciencia e Ingeniería, vol. 11, $\mathrm{n}^{\circ}$ 21, pp. 56-65, 2017

[20] Medeiros, A. C., Freitas, K. R, da Silva, I. D., de Oliveira, M., Guimarães, R. M. y Mendonça, N. M. "Quality index of the surface water of Amazonian rivers in industrial areas in Pará, Brazil," Marine Pollution Bulletin, vol. 123, $\mathrm{n}^{\circ}$ 1-2, pp. 156-164, 2017.

[21] Jacobs, H. L., Gabrielson, I. N., Horton, R. K., Lyon,W. A., Hubbard, E. C. y McCallum, G. E. "Water quality criteria stream vs. effluent standards: An index number system for rating water quality," Journal of the Water Pollution Control Federation, vol. 37, n 3, pp. 300-306, 1965.

[22] Brown, R. M., McClelland, N. I., Deininger, R. A. y Tozer, R. G. "A water quality index-do we dare?," Water Sewage Works, vol. 117, $\quad \mathrm{n}^{\circ}$ 10, pp. 339-343, 1970.

[23] Said, A., Stevens, D. K. y Sehlke, G. "An innovative index for evaluating water quality in streams," Environmental Assessment, vol. 34, n 3, pp. 406-414, 2004.

[24] De Bustamante, I. "Aspectos metodológicos en estudios de calidad del agua," Henares: Revista de Geología, vol. 3, pp. 25-36, 1989.

[25] De Bustamante, I., Sanz, J., Goy, J. L., González,F. M., Encabo, J. L. y Mateos, J. "Estudio de la calidad de las aguas superficiales en los espacios naturales del sur de las provincias de Salamanca y Ávila. Aplicaciones del índice ISQA," Geogaceta, vol. 31, pp. 115-118, 2002.

[26] Martínez, A. M., Goy y Goy, J. L., De Bustamante, I. y Cardeña, C. Z. "Characterization of environmental impact on resources, using strategic assessment of environmental impact and management of natural spaces of "Las Batuecas-Sierra de Francia" and "Quilamas" (Salamanca, Spain)," Environmental Earth Sciences, vol. 71, nº 1, pp. 39-51, 2013.

[27] Alonso, J. "Evaluación de la calidad de las aguas de los arroyos Ca'i Puente y Satĩ de Coronel Bogado con macro invertebrados como bioindicadores," Revista sobre Estudios e Investigaciones del Saber Académico, vol. 9, n 9, pp. 7-12, 2015.

[28] Pacheco,V., Servin, M. R., Valazquez, J. y Servin, M. A. "Situacion actual de la calidad del sub ambalse Mbói Caé de la ciudad de Encarnacion mediante el empleo del Indice Simplificado de Calidad de
Agua (ISQA)," Congreso Internacional de aguas, Ambiente y Energia 2017. Mendoza, Argentina, 2017. Disponible: https://cvl.bdigital.uncu.edu.ar/9733

[29] Méndez, S. A. "Determinación de la influencia de las características fisicoquímicas medidas a través del índice simplificado de calidad del agua (ISQA), sobre la biota medida a través del índice biótico BMWP en la microcuenca del Río Contreras del municipio de Guatemala," Tesis de licenciatura dirigida por J. M. Estrada-Asturias, Facultad de Ingeniería, Universidad de San Carlos de Guatemala, Guatemala, 2015.

[30] M. A. Hernández, M. A. Depuración de Aguas Residuales, Madrid: Paraninfo S.A. España, 1988.

[31] Cubillo, F. "Situación actual de la calidad de las aguas en los ríos de la Comunidad de Madrid (Enero 1986)," Consejería de Obras Públicas y Transportes. Dirección General de Recursos Hidráulicos, Comunidad de Madrid, España, 1986.

[32] Castellanos, C., Valbuena, R. D., Gualtero, D. M. y Ángel, V. J. "Ecosistemas acuáticos de la cuenca alta del río Magdalena", Neiva, Colombia: Universidad Surcolombiana, EMGESA S. A. ESP, 2017.

[33] IDEAM, "Toma de muestras de agua superficiales para la red de calidad del IDEAM,” Bogotá, 14 julio 2017. [En línea]. Disponible: http://sgi.ideam.gov.co/documents/412030/35488871/M-S-LCI004+INSTRUCTIVO+TOMA+DE+MUESTRAS+DE+AGUAS+SUP ERFICIALES+PARA+LA+RED+DE+CALIDAD+DEL+IDEAM.pdf/3 59abf07-33e5-4262-911c-df2e9d6be323?version=1.0. Último acceso 15 julio 2020.

[34] IDEAM, "Determinación de oxígeno disuelto por el método electrométrico," Bogotá, 22 noviembre 2007. [En línea]. Disponible: http://www.ideam.gov.co/documents/14691/38155/Ox\%C3\%ADgeno+ Disuelto+por+Electrometr\%C3\%ADa.pdf/9d532efc-805a-4561-94dba82649af5f91. Último acceso 15 julio 2020.

[35] IDEAM, "pH en agua por electrometría," Bogotá, 28 septiembre 2007. [En línea]. Disponible: http://www.ideam.gov.co/documents/14691/38155/pH+en+agua+por+E lectrometr\%C3\%ADa.pdf/ec53b64e-91eb-44c1-befe-41fcfccdfff1 Útimo acceso 15 julio 2020 .

[36] IDEAM, "Conductividad eléctrica por el método electrométrico en aguas,", Bogotá, 12 julio 2006. [En línea]. Disponible: http://www.ideam.gov.co/documents/14691/38155/Conductividad+El\% C3\%A9ctrica.pdf/f25e2275-39b2-4381-8a35-97c23d7e8af4. Último acceso 15 Julio 2020.

[37] IDEAM, "Demanda de química de oxígeno por reflujo cerrado," Bogotá, 28 diciembre 2007. [En línea]. Disponible: http://www.ideam.gov.co/documents/14691/38155/Demanda+Qu\%C3 \%ADmica+de+Ox\%C3\%ADgeno..pdf/20030922-4f81-4e8f-841cc124b9ab5adb. Último acceso 15 julio 2020.

[38] Baird, R. B., Eaton, A. D. y Rice, E. W. Standard Methods for the examination of water and wastewater, Washington: American Public Health Association, 2017.

[39] IDEAM, "Sólidos suspendidos totales en agua secados a 103-105 ${ }^{\circ} \mathrm{C}$," Bogotá, 02 agosto 2007. [En línea]. Disponible: http://www.ideam.gov.co/documents/14691/38155/S\%C3\%B3lidos+Su spendidos+Totales+en+aguas.pdf/f02b4c7f-5b8b-4b0a-803a1958aac1179c. Último acceso 15 julio 2020.

[40] IDEAM, "Demanda bioquímica de oxígeno 5 días, incubación y electrometría," Bogotá, 04 junio 2007. [En línea]. Disponible: http://www.ideam.gov.co/documents/14691/38155/Demanda+Bioqu\%C 3\%ADmica+de+Ox\%C3\%ADgeno..pdf/ca6e1594-4217-4aa3-9627d60e5c077dfa. Último acceso 15 julio 2020.

[41] IDEAM, "Tiempo y Clima: Boletín climatológico mensual," Bogotá, 2017. [En línea]. Disponible: http://www.ideam.gov.co/web/tiempo-yclima/climatologico-mensual//document_library_display/xYvlPc4uxk1Y/view/18512937. acceso 15 julio 2019

[42] Vásquez, D., H. Silva, H., Angulo, U. y Montañez, M. "Diseño y evaluación de un prototipo de sistema piscícola en San Clemente de Térapa, Moctezuma, Sonora," Ra Ximhai, vol. 7, n 2, pp. 173-185, 2011.

[43] Roldán, G. y Ramirez, J. J. "Fundamentos de limnología neotropical”, Editorial Universidad de Antioquia, 2008.

[44] MacKereth, F. J. H., Heron, J. y Talling, J. F. "Water analysis: some revised methods for limnologist," The Quarterly Review of Biology Washington: Freshwater Biological, vol 54, n 2, pp. 203, 1979.

[45] Abarca, F.J. "Técnicas para evaluacióny monitoreo del estado de los humedales y otros ecosistemas acuáticos," de Perspectivas sobre 
conservación de ecosistemas acuáticos en México, México, Instituto Nacional de Ecología, 2007, pp. 113-144.

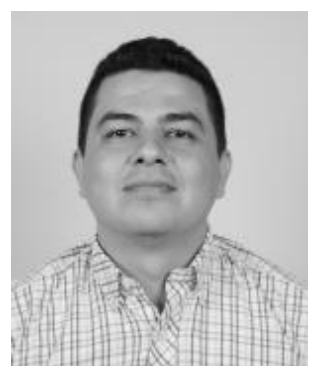

Luis Carlos Losada Benavides. Nacido en Pitalito, Huila (Colombia), el 19 de noviembre de 1984, egresado del programa de Química de la Universidad del Cauca, Magister en Hidrología y Gestión de los Recursos Hídricos de la Universidad de Alcalá y Rey Juan Carlos en España, cuenta con varias publicaciones a la fecha, entre ellas, cuatro artículos internacionales y un capítulo de libro resultado de investigación. Ha participado como ponente en tres eventos internacionales y tres nacionales, posee nueve años de experiencia docente en la Corporación Universitaria del Huila Corhuila, en la cual se desempeñó como director del programa de Ingeniería Ambiental; actualmente es líder del grupo de investigación Efecto Ambiental. ORCID: https://orcid.org/0000-0003-28006327.

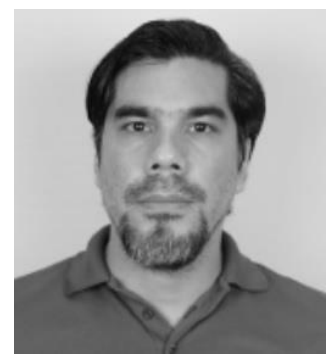

Carlos Alberto Rueda Sanabria. Nacido en Bucaramanga, Santander (Colombia), el 27 de septiembre de 1977, egresado de la escuela de Química de la Universidad Industrial de Santander (2012), Magister en Ciencias Químicas de la Universidad Nacional Autónoma de México (2017). Es docente en la Corporación Universitaria del Huila Corhuila, desde Julio del 2013. Trabajó en el estudio de aceites esenciales de plantas endémicas de Santander desde el punto de vista termodinámico, y análisis químico por GC-FID y GC-MS. También ha trabajado en el análisis químico de aguas. Actualmente realiza investigación en estudio de proteínas y biotecnología. ORDID: https://orcid.org/0000-00021870-9099

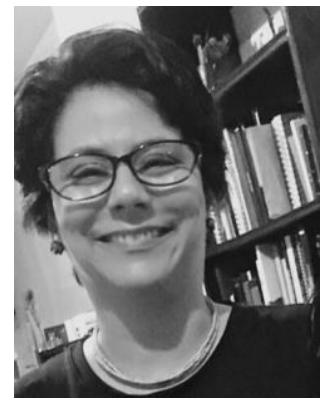

Paula Martínez Silva. Se graduó de Biología en la Universidad Nacional de Colombia en el año 2004 y terminó su maestría en Ecología en la misma Universidad en el año 2007.

Docente Universitaria de tiempo completo en la Corporación Universitaria del Huila Corhuila desde Julio del 2013, donde está a cargo de un semillero que desarrolla investigaciones principalmente en temas relacionado con calidad de agua y aire, producción más limpia y restauración ecológica.

ORCID: https://orcid.org/0000-0002-58727945 